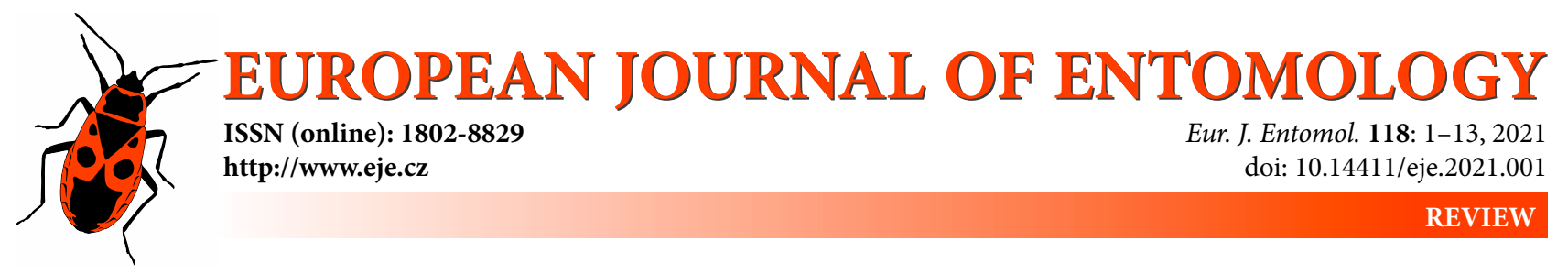

\title{
Insect photoperiodism: Bünning's hypothesis, the history and development of an idea
}

\section{DAVID SAUNDERS}

21, Leadervale Road, Edinburgh EH16 6PB, Scotland, United Kingdom; e-mail: david59.saunders@mypostoffice.co.uk

Key words. Insects, photoperiodism, diapause, Bünning's hypothesis, clock models

Abstract. In insects, the photoperiodic system comprises a linked sequence of events from photoreception to final seasonallyappropriate phenotypes such as overwintering diapause. The first and last of these events are reasonably well known, but central phenomena such as those distinguishing short from long days (time measurement) and the nature, accumulation and transfer of this information through development, metamorphosis and sometimes across generations remains obscure. Bünning's intuitive suggestion that photoperiodic time measurement was a function of the circadian system, made eight decades ago, however, has provided a framework for numerous studies investigating these connections. This review examines the development of Bünning's hypothesis from its origin in plants to the physiology of diapause in insects. Despite considerable inter-species differences, a close and probably causal relationship between circadian rhythmicity and photoperiodism is indicated.

\section{INTRODUCTION}

In 1936, the German plant physiologist Erwin Bünning suggested that time measurement inherent in the photoperiodic phenomenon was a function of the circadian system (Bünning, 1936). The importance of photoperiodism had been appreciated since Garner \& Allard (1920) showed that many plants could only flower and fruit when daylength fell within certain limits, some responding to long days, others to short. These observations were quickly followed by studies on seasonal morph determination in aphids (Marcovitch, 1923, 1924), migration in birds (Rowan, 1926), seasonal breeding cycles in mammals (Baker \& Ranson, 1932) and control of diapause in the silkworm (Kogure, 1933). The selective advantages provided by these phenomena were immediately apparent. The function of circadian rhythmicity, however - although known to control daily up-and-down movements of plant leaves since the 18th century (De Mairan, 1729; Zinn, 1759) - remained more obscure. Charles \& Francis Darwin in their book $O n$ the Power of Movement in Plants, for example, recognised that leaf movements were heritable and must therefore be of importance to the plants concerned (Darwin \& Darwin, 1880), but could not suggest a selective advantage for such a phenomenon. Bünning's insightful suggestion that photoperiodism and circadian rhythmicity were somehow related seemed to provide such a function.

First attempts to test Bünning's hypothesis were wholeplant and whole-animal experiments, many of which were designed to compare properties of the photoperiodic responses of organisms with the canonical features of cir- cadian rhythmicity. This approach revealed many parallels between the two time-measuring systems, but progress remained slow, mainly because the fundamental nature of circadian rhythmicity itself was largely unknown. In insects this situation began to change following the identification of the circadian 'clock' gene period by Konopka \& Benzer (1971) and the subsequent elucidation of the molecular genetics of circadian rhythmicity, particularly for Drosophila melanogaster (e.g. Hall, 2003; Hardin, 2005). Further delay then occurred, however, because D. melanogaster was shown to possess a form of dormancy more like a non-photoperiodic quiescence (Saunders, 2020), although it showed some diapause-like characteristics. Only within the last few years has the extensive repertoire of genetic techniques formerly confined to D. melanogaster been extended to non-Drosophila species showing both circadian rhythmicity and robust photoperiodic diapause responses. Foremost among these techniques has been RNA-interference ('gene silencing') and - with the prospect of CRISPR or 'gene editing' - such technologies should provide the means to address fundamental relationships between the circadian system and seasonal photoperiodism. This review examines the history of Bünning's hypothesis, initially in plants and then in insects, over the eight decades or so since 1936 when it was first proposed. The role of circadian 'clock' genes in insect photoperiodism is not discussed in detail having been reviewed extensively elsewhere (Saunders \& Bertossa, 2011; Goto, 2013; Saunders, 2020). 


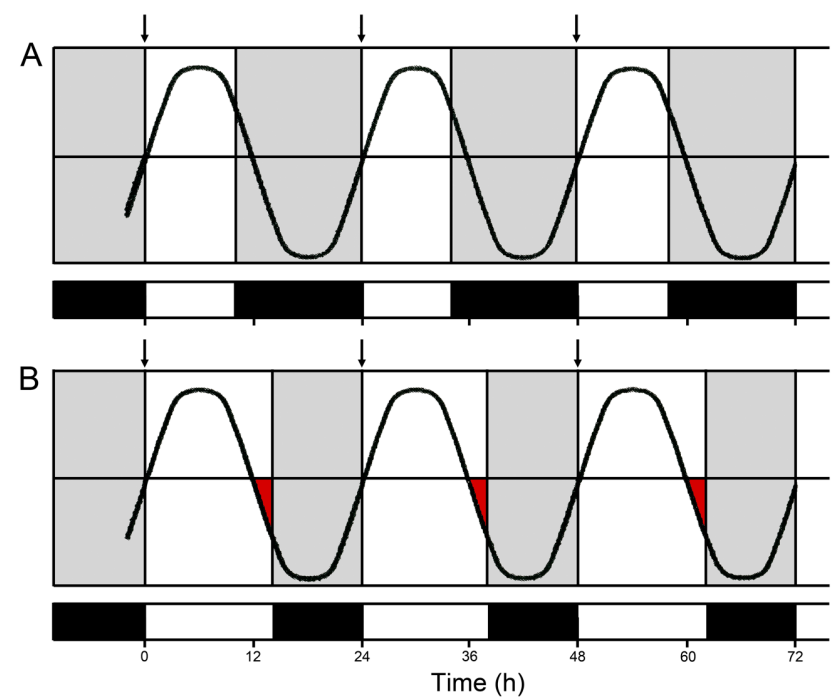

Fig. 1. Bünning's hypothesis (1936) for the involvement of circadian rhythmicity in photoperiodic time measurement (schematic). A - the circadian oscillation in a short day (or long night) autumnal cycle of 10L: 14D; B - the oscillation in a long-day (or short-night) summer cycle of $16 \mathrm{~L}: 8 \mathrm{D}$. That portion of the oscillation above the midline represents the 'photophil' half-cycle; that below the midline represents the 'scotophil'. The small vertical arrows mark the constant phase of the oscillation at 'dawn'; the red areas in B show light encroaching into the 'scotophil' half-cycle to produce long-day effects. Redrawn after Bünning (1960).

\section{Early experiments with plants and insects}

After receiving his doctorate from the University of Göttingen in 1929, Bünning accepted an assistantship in Jena and then, during the 1930s and up to the end of the war in 1945, occupied positions at the Universities of Königsberg, Strasbourg and Cologne before settling permanently in Tübingen from 1946 (see Chandrashekaran, 2006). Although he proposed his model for photoperiodic time measurement as early as 1936, a more explicit version was only later revealed at the Cold Spring Harbor symposium on Biological Clocks in 1960. This version of the model (Fig. 1) - specifically for plant photoperiodism - shows a circadian oscillation entrained by 'short day' (10L : 14D) and 'long day' (16L : 8D) light-dark cycles. In this model Bünning $(1936,1960)$ proposed that the 24 -h period of the oscillation comprised two half-cycles, a 12-h 'photophil' or light requiring section and a 12-h 'scotophil' or dark requiring section. Short-day effects were produced when the light was restricted to the photophil (i.e. in 10L : 14D) but long-day effects when light extended into the scotophil (i.e. in $16 \mathrm{~L}: 8 \mathrm{D})$. For plants, therefore, his model proposed that the circadian oscillation was phase-set at dawn, measured daylength and included a particular light-sensitive phase early in the night which, when illuminated, produced longday effects. Light, therefore, played two roles: entrainment and photoinduction.

\subsection{Night interruption experiments and the Bünsow protocol}

In the immediate post-war years Bünning and his colleagues used 'night-interruption' techniques to test the model, with systematic scanning of the 'nights' of extend-

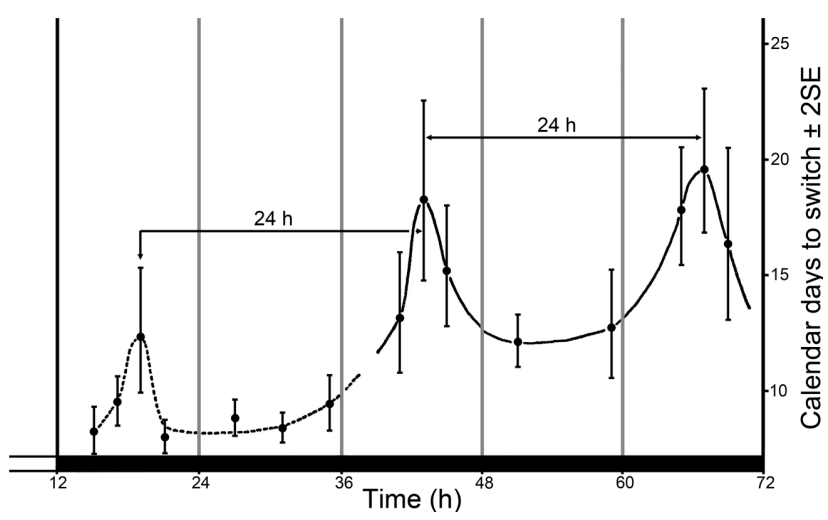

Fig. 2. Nasonia vitripennis. A test of Bünning's hypothesis using the Bünsow experimental protocol in which adult wasps were exposed to a $72 \mathrm{~h}$ light-dark cycle of $12 \mathrm{~L}: 60 \mathrm{D}$ with the $60 \mathrm{~h}$ 'night' interrupted by short $(2 \mathrm{~h})$ light pulses at sequential phases. Peaks of long-day effect (low incidence of diapause in the larval progeny) are produced at about $24 \mathrm{~h}$ intervals in the extended 'night' (at 19, 43 and $67 \mathrm{~h}$ after 'dawn'). Each point represents the mean age at which female wasps 'switch' from the production of nondiapausing to diapausing offspring, a delay in the 'switch-point' representing a long-day effect assumed to mark a phase of light sensitivity. After Saunders (1970).

ed light-dark cycles (i.e. 48 or 72 h) with a short supplementary light pulse designed to locate the presumed lightsensitive phase. In the long-day plant Hyoscyamus niger 2 $\mathrm{h}$ light breaks placed systematically across the 39-h dark phase of a 48-h cycle (9L : 39D) revealed maxima of flower induction $16 \mathrm{~h}$ after dawn and again at $40 \mathrm{~h}$, the two peaks being about $24 \mathrm{~h}$ apart, an interval equivalent to a circadian period ( $\tau$ h) (Claes \& Lang, 1947). Similar results were obtained for the plant Kalanchoë blossfeldiana in $48 \mathrm{~h}$ and 72 h cycles (Bünsow, 1953; Melchers, 1956). Meanwhile, at the University of California at Los Angeles, Hamner (1960), working with the Biloxi variety of soybean maintained in a 72 h cycle of $8 \mathrm{~L}: 64 \mathrm{D}$, showed that flowering was induced when the scanning pulse fell 16, 40 and $64 \mathrm{~h}$ after dawn - again at $24 \mathrm{~h}$ intervals. This technique, clearly showing circadian involvement in photoperiodic induction, became known as the Bünsow protocol.

Among the insects, Bünsow responses were first clearly recorded in the parasitic wasp Nasonia vitripennis (Saunders, 1970) (Fig. 2). In this species, larval diapause occurs just before pupation but is induced by photoperiod experienced in the maternal generation (Saunders, 1965, 1966). Female wasps exposed to long days lay eggs within the puparia of their blow fly hosts which give rise to nondiapausing or continuously-developing progeny. Those exposed to the short days of autumn, however, produce nondiapausing progeny for the first few days before switching abruptly to the production of larvae entering diapause. Wasps exposed to 2-h light breaks in a 48-h cycle (12L : 36D) (Saunders, 1970) showed peaks of long-day effect (delayed switching) when the scanning pulses fell 19 and $43 \mathrm{~h}$ after dawn, 24 or $\tau \mathrm{h}$ apart. In a $72-\mathrm{h}$ cycle (12L:60D) peaks of long-day effect occurred 19, 43 and $67 \mathrm{~h}$, again $\tau$ h apart (Fig. 2). These results constituted 'positive' Bünsow effects, consistent with circadian involvement in the photoperiodic phenomenon. 

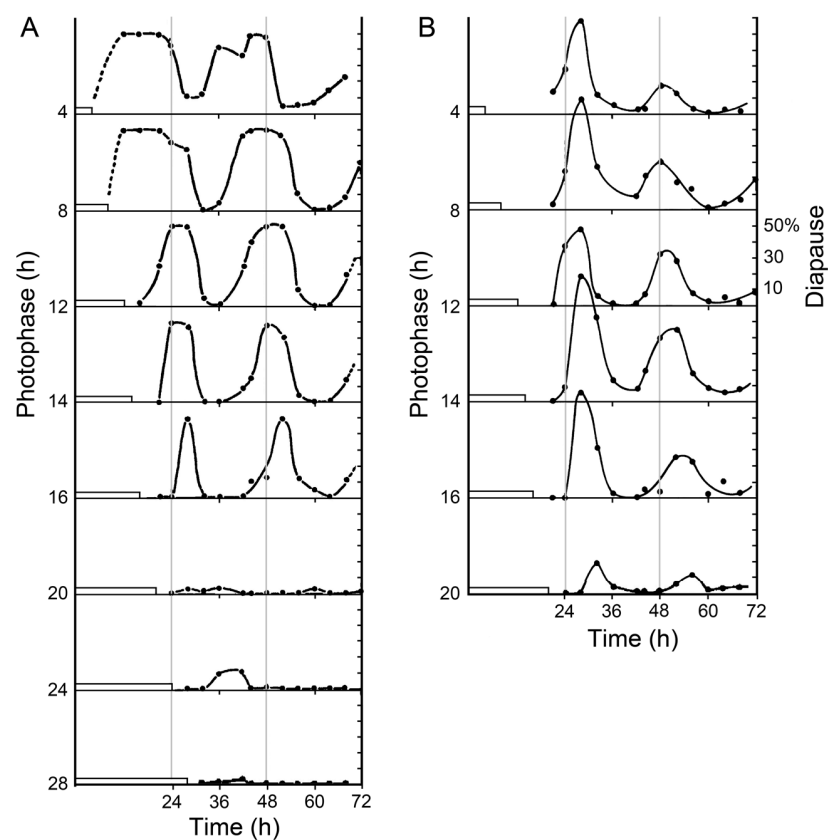

Fig. 3. Results of Nanda-Hamner experiments. In panel A, adults of Nasonia vitripennis were exposed, at $17^{\circ} \mathrm{C}$, to light cycles containing a fixed photophase (4 to $28 \mathrm{~h}$ ) coupled to a range of dark periods to give overall cycles lengths up to $72 \mathrm{~h}$. Host Sarcophaga pupae were provided daily and the incidence of Nasonia larval diapause ascertained on days 14 and 15 of adult life, an age at which the 'switch' to diapause progeny production under short days (long nights) was completed. In panel B, larvae of S. argyrostoma were exposed, at $17^{\circ} \mathrm{C}$, to similarly extended light cycles and the resulting pupae assessed for diapause or nondiapause development. In both species peaks of high diapause incidence were observed at roughly $24 \mathrm{~h}$ intervals as the period of the light cycle was extended, providing evidence for the circadian involvement in photoperiodic time measurement (see text for further details). After Saunders (1973a, 1974).

'Positive' Bünsow results from scanning light pulses in 48-h and 72-h cycles have now been recorded in at least 10 other species from five insect orders (Orthoptera, Homoptera, Lepidoptera, Diptera and Hymenoptera) and also from mites (Saunders, 2002 p. 346). In some other cases, however, results have proved 'negative' for circadian effects; these results will be discussed further in section 6 below.

\subsection{Systematic lengthening of the night phase in non-24 h cycles: The Nanda-Hamner protocol}

A second test for the involvement of the circadian system in plant photoperiodism was devised by K.C. Hamner and his associates (Blaney \& Hamner, 1957; Nanda \& Hamner, 1958; Hamner, 1960; Takimoto \& Hamner, 1964) and later for birds (W.M. Hamner, 1963); this became known as the Nanda-Hamner protocol. In this type of experiment organisms were exposed to a range of light-dark cycles containing a fixed photophase but a variable period of darkness; e.g. a 16-h cycle (8L: 8D) to a cycle of $72 \mathrm{~h}(8 \mathrm{~L}: 64 \mathrm{D})$ or more, covering several multiples of an endogenous circadian period. Results for plants and birds showed periodic maxima of induction in cycles close to 24,48 and $72 \mathrm{~h}$ in length, but minima of induction in cycles close to 36 or $60 \mathrm{~h}$.
'Positive' Nanda-Hamner (NH) responses such as these were demonstrated in insects for Nasonia vitripennis (Saunders, 1968, 1974) using cycles ranging from 12 to 72 $\mathrm{h}$ in length; the photophase was also varied in each cycle from 4 to $28 \mathrm{~h}$ (Fig. 3a). In these experiments, progeny from wasps on days 14 to 15 of adult life were assessed for diapause. At this age wasps exposed to 'strong' short days had all switched to the production of diapausing larvae, whereas those exposed to long days continued to produce developing progeny (Saunders, 1965, 1966), the 14 to 15 day 'slice' thereby providing an accurate and convenient assessment of the photoperiodic response. Results of these NH experiments (Fig. 3a) showed a high incidence of larval diapause when the adults were exposed to cycles close to 24,48 and $72 \mathrm{~h}$ in length, but a low incidence of diapause in cycles close to 36 and $60 \mathrm{~h}$. For the flesh fly Sarcophaga argyrostoma (Saunders, 1973a), NH experiments produced a similar circadian periodicity (Fig. 3b). Results for both species are examined more closely in later sections.

In other insects, 'positive' Nanda-Hamner results have now been recorded in at least 12 species from 6 orders (Orthoptera, Homoptera, Coleoptera, Lepidoptera, Diptera and Hymenoptera) and from two species of Acarina (Saunders, 2002, pp. 352-353). 'Negative' Nanda-Hamner results have also been recorded; these will be discussed later in section 6 .

\section{Drosophila pseudoobscura: Pittendrigh's model for the photoperiodic clock}

Working with the pink boll worm moth Pectinophora gossypiella, Adkisson $(1964,1966)$ used the night interruption technique to systematically probe the dark phases of a range of 24-h light-dark cycles (6L : 18D to 13L : 11D) with $1 \mathrm{~h}$ pulses of light. This procedure produced not one, but two discrete phases of diapause inhibition, one (point A) early in the night and another (point B) late in the night. This unexpected result raised important questions: were there two light-sensitive (photoinducible) phases in Bünning's scotophil or, if there was only one, was it at A or B? Pittendrigh (1966) solved this apparent conundrum by comparing Adkisson's results with his own observations on the entrainment of the pupal eclosion rhythm of Drosophila pseudoobscura to 'skeleton' photoperiods and used these observations as a basis of a model for the photoperiodic clock - even though this species was apparently 'day neutral' without a photoperiodic, or a diapause, response.

Pittendrigh (1966) made four important observations: (1) that the eclosion oscillator of D. pseudoobscura achieved steady-state entrainment to the combination of main light phase and the scanning pulse, which he called an 'asymmetrical skeleton' photoperiod. He also showed, however, (2) that the eclosion oscillator damped out in extended periods of light but recommenced, upon transfer to darkness, at a particular phase equivalent to the start of the subjective night (at a phase called Circadian Time, CT $12 \mathrm{~h}$ ). Furthermore, (3) he stressed that whenever light impinged upon a circadian oscillation it caused a phase shift, either a delay or an advance. Therefore, in the version of Bünning's model that he now proposed (Fig. 4), the photoperi- 


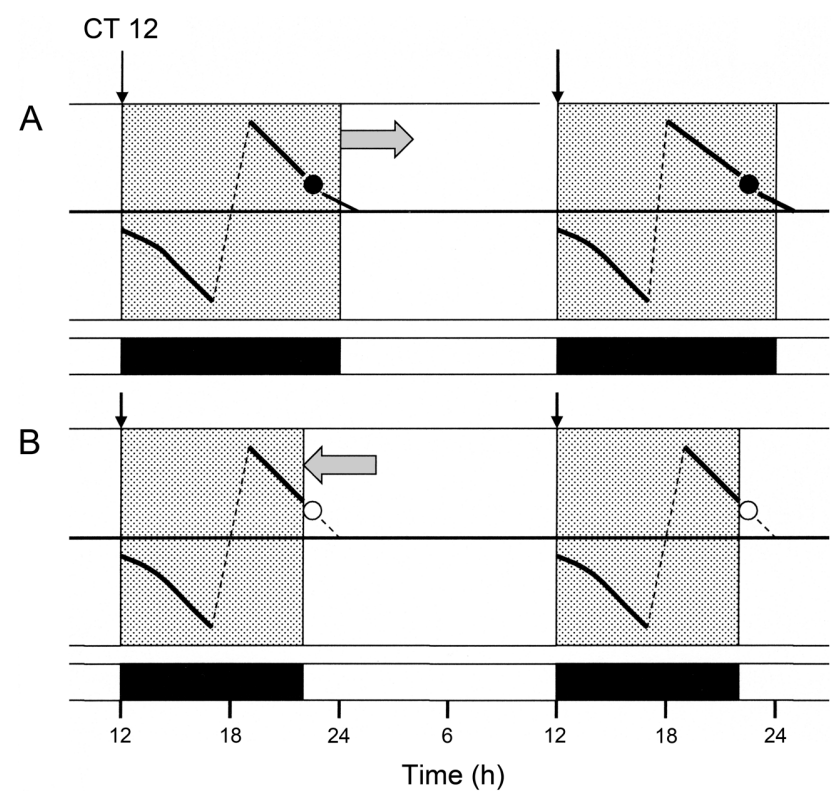

Fig. 4. Pittendrigh's external coincidence model, appropriate for insects such as Sarcophaga argyrostoma (and probably widespread among other insects) that 'measure' night length (schematic). The oscillation, shown here as a Type 0 high amplitude phase response curve (Winfree, 1970) is phase-set to CT 12 at the light-to-dark transition and depicts phase delays below the midline and phase advances above it. In (A), an autumnal long-night cycle (12L: 12D), the photoinducible phase $\left(\varphi_{i}\right)$ occurring about $9.5 \mathrm{~h}$ after dusk, falls in the dark $(\bullet)$ and diapause supervenes. In (B) a summer short-night cycle (16L: 8D) $\varphi_{\mathrm{i}}$ (o) is illuminated by dawn light, leading to nondiapause development. Large horizontal arrows show the 'movement' of dawn in relation to the oscillation as seasons change. Redrawn after Pittendrigh (1966).

odic oscillator was shown in the form of a phase response curve (PRC) (Winfree, 1970). But (4), since the photoperiodic oscillator is at a fixed phase (CT 12) at light-off, time measurement must begin at 'dusk'. Consequently (in most cases) the clock - in contrast to Bünning's original proposal - measures nightlength rather than daylength. This suggests that the light-sensitive or 'photoinducible' phase (designated as $\varphi_{\mathrm{i}}$ ) must occur at the end of a critical nightlength $(\mathrm{CNL})$ - or at point B. A light pulse falling at point A merely causes a phase delay of $\varphi_{i}$ into the following main light component thereby causing nondiapause development, whereas a light pulse falling late in the night causes a phase advance until, at point $\mathrm{B}$, it coincides directly with $\varphi_{\mathrm{i}}$, again leading to nondiapause development. This modified version of Bünning's original model is more appropriate for S. argyrostoma than that depicted in Fig. 1 and may be also be appropriate for other insects.

\section{The relative importance of the light and dark phases: Circadian surfaces}

Numerous studies in which the light (L) and dark (D) components of the daily cycle were independently varied have indicated that the duration of the night is frequently more important in insects than the duration of the day (Saunders, 2013) - although some species, such as the linden bug Pyrrhocoris apterus may 'measure' daylength (Saunders, 1987a). In the flesh fly S. argyrostoma, for ex-

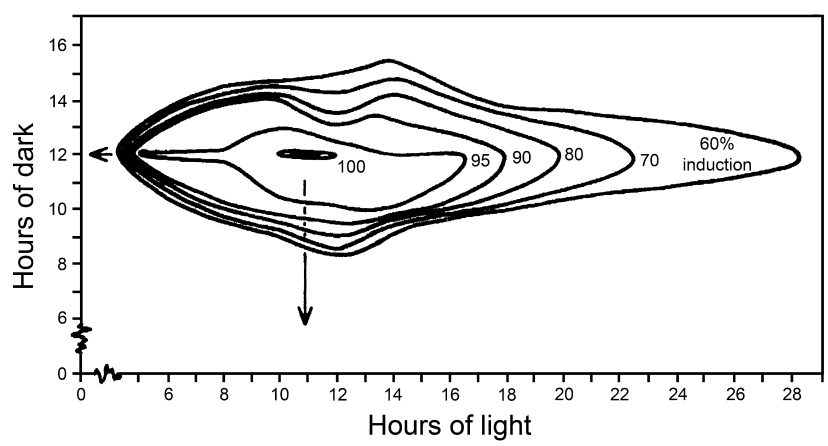

Fig. 5. Larval diapause induction in the European corn borer moth Ostrinia nubilalis. A surface of 'isoinduction lines', based on data from Beck (1962). The lines, or 'contours', connect combinations of light and darkness giving rise to equal levels of diapause. The resulting 'circadian surface' suggests that the duration of darkness is more important than that of the light, and that maximum diapause incidence occurs in 11L:12D, suggesting that a circadian oscillation (period about 23 h) may be involved. From Pittendrigh (1966) after Beck (1962).

ample, the incidence of pupal diapause was very low in cycles containing a short night (e.g. 12L : 8D and 16L : 8D) but approached $100 \%$ in cycles containing a long night (e.g. 12L:12D and 16L:12D), almost regardless of the duration of L (Saunders, 1973a). Beck (1962) studied the induction of larval diapause in the European corn borer $O s$ trinia nubilalis raised in cycles containing D values of 10 , 12 or $14 \mathrm{~h}$ combined with a wider range of $\mathrm{L}$. He found the highest incidence of diapause $(90 \%$ or over) when $\mathrm{D}$ was within this narrow range. Maximum diapause induction also occurred when $\mathrm{L}+\mathrm{D}$ was close to the period of the presumed circadian oscillator.

The relative importance of $\mathrm{L}$ and $\mathrm{D}$ for larval diapause induction in $O$. nubilalis was later indicated in a 'circadian surface' calculated by Pittendrigh (1972) from Beck's data in which diapause incidence was plotted against the lengths of $\mathrm{D}$ and of $\mathrm{L}$ in a three-dimensional plot with points of equal diapause incidence presented as 'contours'. This type of plot (Fig. 5) clearly showed a central 'mountain' of high diapause incidence peaking close to $11 \mathrm{~L}: 12 \mathrm{D}$ - a period of $23 \mathrm{~h}$, equivalent to that of a circadian oscillator that may underlie the response. Although these data were not extended to cover longer cycle lengths, as in the Nanda-Hamner protocol, Pittendrigh predicted that further 'mountains' of high diapause incidence should occur at circadian intervals as the D axis was extended.

Extended plots of this type were later produced for $S$. argyrostoma and N. vitripennis (Saunders, 1973a, 1974). In the flesh fly (Fig. 6A), two 'mountains' of high diapause incidence were revealed, about $24 \mathrm{~h}$ apart. Moreover, in lighting regimes containing a short fixed photophase, diapause maxima occurred parallel to light-on, but once the photophase exceeded about $12 \mathrm{~h}$ the maxima followed light-off (Saunders, 1973a) because the rhythm is re-initiated at the light-dark transition at a constant phase equivalent to that at the time of the light-dark transition in a cycle of $12 \mathrm{~L}: 12 \mathrm{D}$. This behaviour is reminiscent of that shown by the pupal eclosion rhythm of $D$. pseudoobscura in constant darkness after a last photophase longer than about 12 

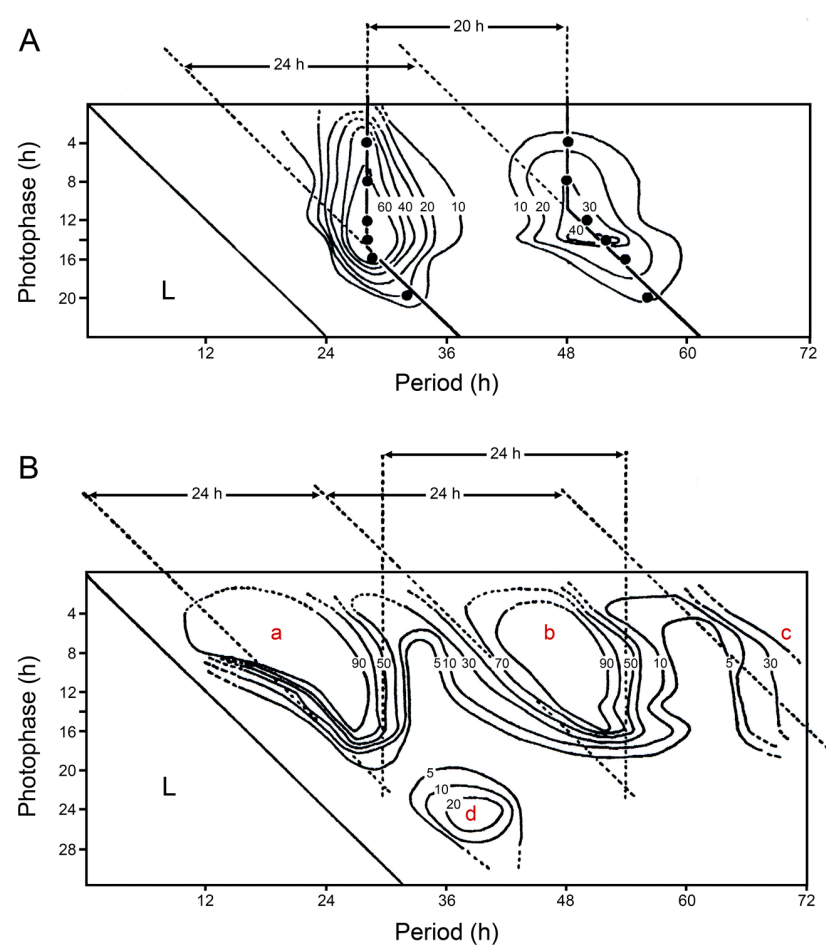

Fig. 6. Isoinduction circadian surfaces derived from Nanda-Hamner experiments (data shown in Fig. 3). In A - Sarcophaga argyrostoma, two 'mountains' of high diapause incidence in the extended night are shown, peak incidence parallel to dawn after a short photophase but parallel to dusk once the photophase becomes longer that about $12 \mathrm{~h}$. In B - Nasonia vitripennis, three principal 'mountains' ( $a, b$ and c) are evident, the ascending slopes of which appear to lie parallel to dusk and the descending slopes parallel to dawn; a fourth 'mountain' (d) is also evident after photophases longer than $24 \mathrm{~h}$. These results suggest that significant differences exist between the photoperiodic clocks of the two species; these differences are addressed in the text. $L$ shows the light component of each cycle as a 'light wedge'. Data from Saunders $(1973,1974)$.

h (Pittendrigh, 1966) - and also in S. argyrostoma (Peterson \& Saunders, 1980) - and confirms earlier observations that nightlength measurement commences at 'dusk' after longer photophases. Such behaviour might suggest a slow accumulation of photons during the photophase, reaching a threshold after about $12 \mathrm{~h}$ of illumination. The circadian surface obtained for $N$. vitripennis (Fig. 6B) shows a more complex pattern of diapause maxima in extended nightlengths (Saunders, 1974); this response pattern will be considered further in section 8 .

\section{Three models for the photoperiodic clock}

The photoperiodic clock model shown in Fig. 4 is appropriate for $S$. argyrostoma, but comparative studies have revealed substantial differences in other species. In order to account for such differences, Pittendrigh (1972) proposed three versions of Bünning's general hypothesis: (1) External Coincidence which - like Bünning's original proposition - consisted of a single circadian oscillation, phaseset (entrained) by the light-dark cycle in such a way that a light-sensitive (or 'photoinducible' phase, $\varphi_{\mathrm{i}}$ ) occurred at point $\mathrm{B}$, the critical nightlength after the onset of darkness, as described above for S. argyrostoma. This model supposed that light had two effects: entrainment and pho- toinduction (of the diapause to nondiapause switch in development). (2) The second model, Internal Coincidence, consisted of two oscillators separately entrained by the 'dawn' and 'dusk' transitions of the daily light-dark cycle, whose internal or mutual phase relationship changed with the length of the photophase, induction of diapause or development occurring according to the 'overlap' between particular phases of the two components. Such a model - previously also envisaged by Russian investigators (Tyshchenko, 1966; Danilevskii et al., 1970) - supposed that light had only one role, that of entrainment. (3) In a third model, or 'Resonance' principle, Pittendrigh supposed that circadian rhythmicity was not necessarily involved in time measurement itself, but at a more downstream level between the clock and the processes it controlled. These interactions could explain 'positive' Nanda-Hamner profiles in which the inductive effect was high in cycles close to the circadian period or multiples thereof (i.e. $\tau$ or modulo $\tau)$, but low when the period of the light-dark cycle was far from $\tau$. Other circadian-based models for the insect photoperiodic clock have been suggested; these are listed by Saunders (2002, p. 397; Vaz Nunes \& Saunders, 1999) but are not considered further.

In contrast to the models outlined above, non-repetitive 'hourglass-like' timers have been proposed, most importantly in the extensive work of A.D. Lees on the photoperiodic regulation of seasonal morphs in the green vetch aphid, Megoura viciae (Lees, 1973). In this insect reproductive mode was thought to be regulated by a non-circadian or linear timer measuring nightlength, the short nights of summer leading to parthenogenetic and viviparous morphs (virginoparae) whereas the long nights of autumn induce the production of males and of females (oviparae) that lay diapausing eggs. Hourglass-like clocks and their relationship to the circadian system are examined in section 6 .

\section{The photoperiodic clock-counter mechanism}

Photoperiodic regulation of insect diapause comprises a linked sequence of events from (1) photoreception, through (2) nightlength or daylength measurement by the photoperiodic clock and (3) accumulation of these events by a 'counter' mechanism to (4) the endocrine effectors of the diapause state. In this review, the second and third of these events will be examined in more detail, particularly the effect of temperature on the proportion of insects entering diapause and the fall in diapause incidence under very short photophases, both of which involve aspects of circadian rhythmicity. Firstly, however, properties of the photoperiodic 'counter' mechanism will be reviewed, with reference to both $N$. vitripennis and $S$. argyrostoma.

\subsection{The photoperiodic counter}

Most insects, having measured the length of the night (or of the day) by the photoperiodic clock, then proceed to accumulate ('add up') the effects of such daily cycles to an internal threshold that triggers diapause or nondiapause development; this constitutes the photoperiodic 'counter' mechanism (Saunders, 1981). In N. vitripennis, for example, wasps exposed to long-night cycles of 12L:12D 


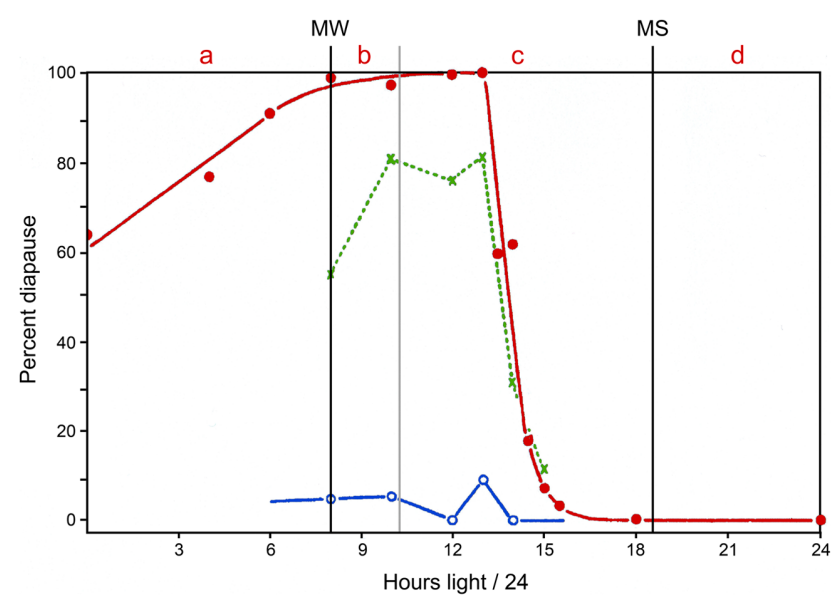

Fig. 7. Sarcophaga argyrostoma. Incidence of pupal diapause in cultures exposed as larvae to photoperiod at $15^{\circ} \mathrm{C}$ (red), $18^{\circ} \mathrm{C}$ (green) and $25^{\circ} \mathrm{C}$ (blue) showing the effect of temperature upon diapause incidence. MW - approximate daylength (including twilight) at mid-winter at $55^{\circ} \mathrm{N}$; MS - ditto at mid-summer. Daylengths in sections a and $d$ are never experienced in nature; section b only occurs in the depth of winter when the insects are already in diapause. Only section $c$ is of ecological importance, containing the critical daylength (CDL) operating the seasonal switch in metabolism from nondiapause to diapause development. Data show (1) reduction in diapause incidence with a rise in temperature under 'strong' short days and (2) reduction in diapause under ultrashort photoperiods (section a). After Saunders (1971).

at temperatures of $15,20,25$ and $30^{\circ} \mathrm{C}$ produced most of their first progeny as continuously-developing individuals but then switched, one by one, to the production of larvae that entered diapause. Although the length of reproductive life and the daily rate of oviposition were both temperature dependent $\left(\mathrm{Q}_{10}\right.$ between 2 and 3), the 'switch points' (or required day numbers, RDNs) of the wasps at the four temperatures showed a high degree of temperature compensation, with a $\mathrm{Q}_{10}$ value of about 1.04 (Saunders, 1966). A similar relationship between temperature and diapause induction was found for pupal diapause induction in the flesh fly S. argyrostoma (Saunders, 1971, 1992) and for larval diapause in the blow fly Calliphora vicina (Saunders, 1987b). Interaction between the temperature-compensated (circadian-related) summation of light cycles and the temperature-dependent length of the photoperiodic 'sensitive period' resulted in a lower incidence of diapause at higher than at lower temperatures.

\subsection{The photoperiodic response curve}

Fig. 7 shows typical photoperiodic response curves (PPRCs) for a mid-latitude species (S. argyrostoma). Vertical lines marked MW and MS show the approximate shortest (mid-winter) and longest (mid-summer) daylengths at about $55^{\circ} \mathrm{N}$, including periods of twilight. The entire range of illumination from continuous darkness (DD) to continuous light (LL) is then divided into four sections. Sections $a$ and $d$ are outside the natural range and $b$ only occurs during the winter when the insects are already in diapause. Only section $c$ is of ecological significance: it is dominated by the abrupt critical daylength (CDL) or critical night- length (CNL) separating the two developmental pathways, diapause or nondiapause.

The fall in diapause incidence in 'ultrashort' days (region $a$ in Fig. 7) cannot be explained by either Bünning's original model (Fig. 1) or by Pittendrigh's version of External Coincidence (Fig. 4) because the presumed photoperiodic oscillator in these models would be expected to persist ('free-run') in extended periods of darkness giving rise to a consistently high diapause incidence. In S. argyrostoma, however, ultra-short photophases of about 1 to $5 \mathrm{~h}$ elicit low amplitude Type 1 phase response curves (PRCs) in which pulses of light elicit only small phase shifts, whereas longer light pulses give high amplitude Type 0 PRCs eliciting much larger phase shifts and more rapid entrainment (see Winfree, 1970; Saunders, 1978a). With very strong short-day pulses (10 to $14 \mathrm{~h}$ ) the Type 0 PRCs become straight lines parallel to the end of the pulse, regardless of the circadian time at its inception, thereby setting the oscillation to CT 12 at light-off, as described above in section 3. Since Type 0 PRCs give large phase shifts, either advance or delay, steady-state entrainment of the photoperiodic oscillator to the light cycle is rapidly accomplished with few transient cycles, facilitating efficient summation by the counter mechanism. Type 1 PRCs, on the other hand, give much smaller phase shifts requiring the photoperiodic oscillator to pass through a greater number of transient cycles before steady state is achieved (Saunders, 1982a), the consequence of which is reduced diapause incidence. An increase in the irradiance of ultra-short photophases applied to $S$. argyrostoma served to increase the amplitude of the phase shifts from Type 1 towards Type 0 (see Winfree, 1970 for the Drosophila case) and resulted in a decrease in the number of transient cycles, thereby increasing the proportion of larvae entering pupal diapause (Saunders, 1982a). Since photoperiodic induction depends in part on the summation of consecutive light-dark cycles by the counter mechanism during the sensitive period (Saunders, 1971) the conclusion is that diapause incidence is affected by the number of transient cycles experienced before steady-state entrainment is attained.

\section{Damping circadian oscillators: The hourglass-oscillator controversy}

'Negative' Bünsow and Nanda-Hamner results (section 1) have been used as evidence that photoperiodic time measurement is not a function of the circadian system, but is product of a linear, non-oscillatory 'hourglass' timer. The most persuasive evidence in favour of this suggestion came from the extensive work of Lees $(1965,1973)$ on the green vetch aphid $M$. viciae, in which short summer nights induced aphids to produce a succession of wingless, viviparous and parthenogenetic generations (virginoparae), whereas long autumnal nights led to the production of males and also winged sexual females (oviparae) laying diapausing eggs. 'Negative' Nanda-Hamner results obtained for $M$. viciae and some other species were in distinct contrast to those showing a circadian involvement in photoperiodic timing and led to a lasting controversy regarding the nature of the insect photoperiodic clock. 
Comparative studies of time measurement, however, revealed many similarities between those showing hourglass-like responses and those considered to use a clock with circadian characteristics. For example, in common with most other insects, nightlength in $M$. viciae occupies a central role in time measurement (Lees, 1965) although extended dark periods are only recorded as a single long night with no evidence of persistent circadian oscillation. Furthermore, night interruption experiments in the aphid using a long night cycle (e.g. in $13.5 \mathrm{~L}: 10.5 \mathrm{D}$ ), with the dark phase systematically scanned by 1 -h light pulses, produced two points of long-day (or short-night effect) as in many other species (section 2), although Lees (1973) interpreted this result as a linked sequence of biochemical reactions rather than the result of photic entrainment of a circadian oscillation. In addition, action spectra (Lees, $1966,1971)$ showed that maximum sensitivity for an interruption early in the night (peak A) was in the blue (450 to $470 \mathrm{~nm}$ ) whereas that for an interruption late in the night (at peak B) showed sensitivity extending from blue into the red end of the spectrum, a result clearly comparable to that for the flesh fly Sarcophaga similis (Goto \& Numata, 2009), a species for which external coincidence is indicated (see section 7). The only consistent difference between the circadian-based and hourglass-like models, therefore, was the presence or absence of persistent oscillation in extended periods of darkness.

Resolution of this divergence of opinion was first provided by Bünning himself $(1960,1969)$ who suggested that the photoperiodic oscillator underwent dampening in extended periods of darkness but was 'boosted' to full amplitude by a train of light pulses. With this interpretation, hourglass-like clocks are best regarded as heavily dampened oscillators, probably having the same molecular components as continuously running systems but persisting at most for only a few cycles unless exposed to light pulses. Data showing such dampening are hard to demonstrate given the covert nature of the photoperiodic oscillator, but experimental evidence compatible with this interpretation is provided for two species of Diptera (Saunders \& Lewis, 1988 ) and theoretical support by a computer model (Lewis \& Saunders, 1987; Saunders \& Lewis, 1987a, b) based on extensive experimentation. Further support for oscillator dampening in photoperiodism is provided in section 7 .

Hourglass-like photoperiodic responses are also apparent in several species of high latitude drosophilids such as Drosophila ezoana (Vaze \& Helfrich-Förster, 2016) and D. montana (Kauranan et al., 2019; Tyukmaeva et al., 2020). In these species lengthening autumnal nightlength is measured by a clock presenting properties of external coincidence but results of Nanda-Hamner experiments fail to show repeated cycles of nightlength measurement in extended darkness. Such results indicate hourglass-like photoperiodic clocks based on heavily dampened oscillators.

Fig. 8 shows what may be expected for this type of clock in Nanda-Hamner experiments. For a mid-latitude species such as $S$. argyrostoma, a 'positive' Nanda-Hamner result occurs with a high incidence of diapause in cycles close

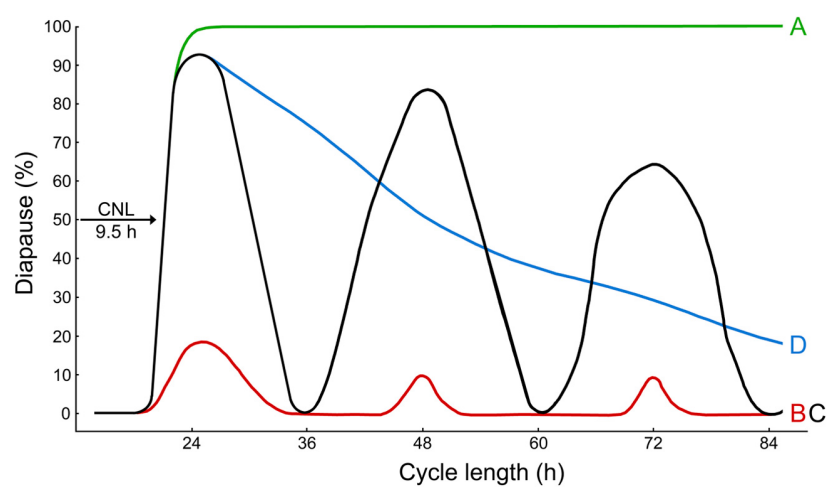

Fig. 8. Schematic representations of Nanda-Hamner experiments in insects with dampening circadian photoperiodic oscillators (external coincidence). Curve A (green) shows the response of a midlatitude insect at low temperature (such as Sarcophaga argyrostoma at $16^{\circ} \mathrm{C}$, Saunders, 1973a) in which diapause incidence is at or near 100 per cent under all cycles longer than $24 \mathrm{~h}$. Curve C (black) shows the periodic response of an insect such as $S$. argyrostoma at moderate temperature $\left(20\right.$ to $\left.22^{\circ} \mathrm{C}\right)$ showing a 'positive' $\mathrm{NH}$ response with peaks of high diapause incidence occurring at circadian intervals as the dark period is extended, the declining amplitude of the three peaks reflecting the dampening of the circadian oscillator. Curve $B$ (red) shows the response at high temperature (S. argyrostoma at $28^{\circ} \mathrm{C}$, Saunders, $1982 \mathrm{~b}$ ) in which diapause incidence under longer dark periods is almost prevented. Curve $D$ (blue) represents the 'negative' or hourglass-like $\mathrm{NH}$ response observed at moderate temperature attributed to the action of a highly dampened photoperiodic oscillator as seen in aphids (Lees, 1986) or some high-latitude drosophilids (see Vaze \& Helfrich-Förster, 2016; Tyukmaeva et al., 2020). In all simulated curves the initial period of darkness from light-off ('dusk') is the critical nightlength (CNL, here $9.5 \mathrm{~h}$ ) measured by the photoperiodic clock according to the principles of external coincidence.

to 24,48 and $72 \mathrm{~h}$ in length, but a low incidence in cycles close to 36 and $60 \mathrm{~h}$. In $S$. argyrostoma, however, this result was only observed at moderate temperature (20 and $22^{\circ} \mathrm{C}$ ) (Fig. 8 , curve $\mathrm{C}$ ): at lower temperature $\left(16^{\circ} \mathrm{C}\right)$ diapause incidence was almost $100 \%$ in all cycle lengths over $24 \mathrm{~h}$, producing an hourglass-like response (Fig. 8, curve A) (data in Saunders, 1973a) very similar to that obtained for $M$. viciae at $15^{\circ} \mathrm{C}$ (Lees, 1973).

The effects of temperature on circadian resonance are complex. For example, at lower temperature, the effect on diapause incidence is three-fold: (1) diapause incidence is directly increased by chilling, (2) the photoperiodic 'sensitive period' (duration of larval development, for example) is increased (Saunders, 1971), but (3) the clock oscillation becomes dampened in amplitude (Lewis \& Saunders, 1987; Saunders \& Lewis, 1987a, b). Therefore, according to the Lewis-Saunders model, the net result is that the oscillation may not reach the threshold above which diapause incidence can be reduced by dawn light, according to the principles of external coincidence. Of course, circadian resonance may occur at $16^{\circ} \mathrm{C}$ but would remain hidden because $100 \%$ diapause is the maximum result possible! Conversely, at higher temperature (Fig. 8, curve B) diapause incidence in cycles over $24 \mathrm{~h}$ in length is almost reduced to zero (as in Saunders, 1982b): although the sensitive period at raised temperature is shortened (Saunders, 1971), the photoperiodic oscillator becomes more sustained (Lewis \& 


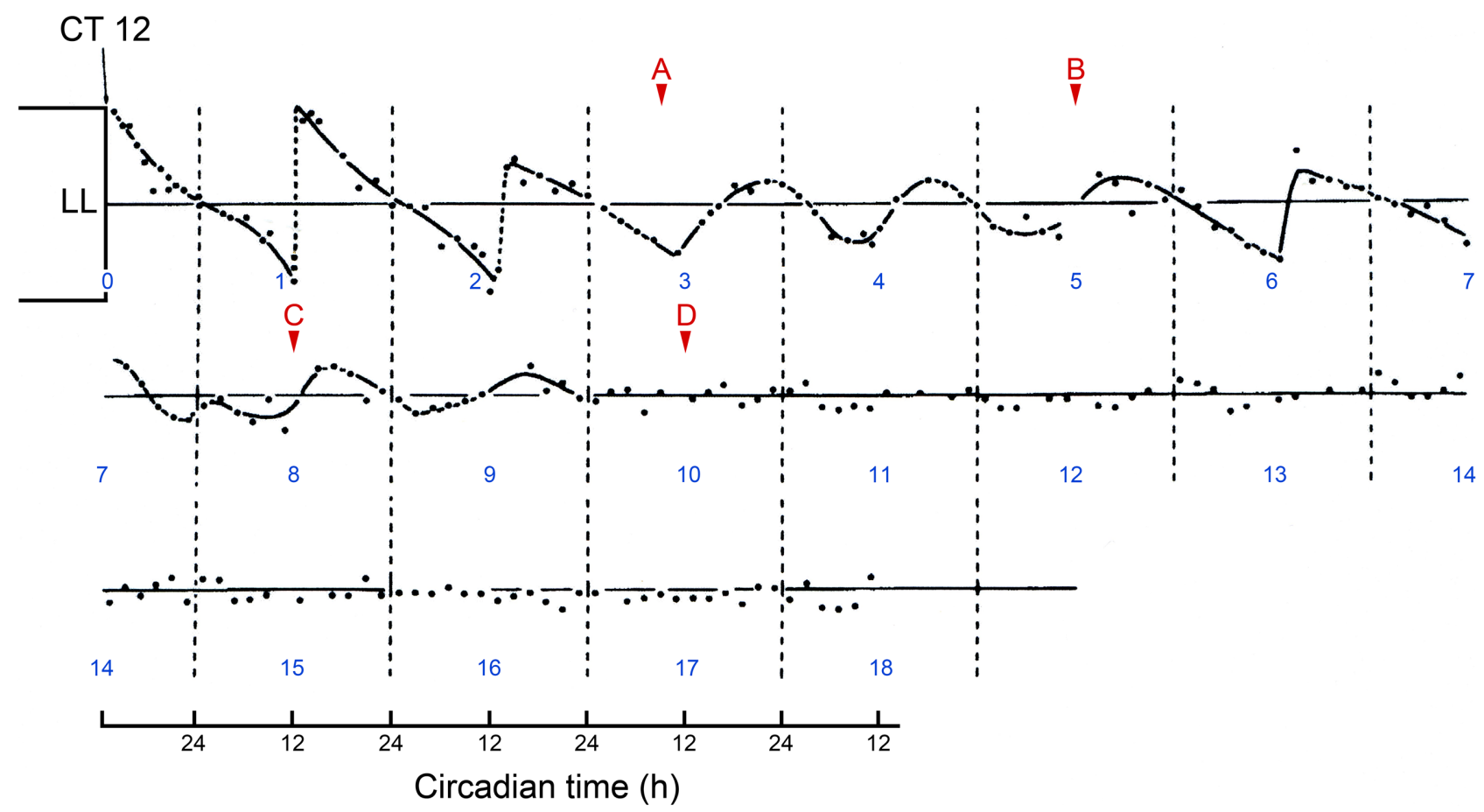

Fig. 9. Sarcophaga argyrostoma. An 'extended phase response curve' (PRC) throughout the larval sensitive period. Newly deposited $1^{\text {st }}$ instar larvae were transferred from constant darkness (LL) to darkness (circadian time, CT $12 \mathrm{~h}$ ) and then exposed to single $12 \mathrm{~h}$ pulses of light at intervals during development. The PRCs obtained were high amplitude Type 0 (Winfree, 1970) during the first two days in darkness but then steadily declined through a series of lower amplitude Type 1 responses until final extinction before the $3^{\text {rd }}$ instar larvae burrow to pupariate. A - time when larvae leave their food; B - mature ( $3^{\text {rd }}$ instar) larvae sieved from sawdust; C to D - time of maximal puparium formation. (From Saunders, 1979a.)

Saunders, 1987) so that the greatly reduced diapause peaks in 48-h and 72-h cycles (Fig. 8 curve B) are less dampened, remaining similar in amplitude (Saunders, 1982b). Further increase in temperature, however, may eliminate diapause in all of these cycles because nothing can be reported below $0 \%$.

In insects such as $M$. viciae and the high-latitude drosophilids, D. ezoana and D. montana that are presumed to have heavily dampened photoperiodic clocks, periodic occurrence of diapause was inapparent even at moderate temperature (Lees, 1986; Vaze \& Helfrich-Förster, 2016; Tyukmaeva et al., 2020). Although diapause incidence in cycles longer than $24 \mathrm{~h}$ was variable, it was random with no obvious periodicity. However, in a 48-h cycle diapause incidence was approximately half of that in a 24-h cycle, and in a 72-h cycle it was approximately one third, reflecting the number of times a boosting light pulse occurred in each cycle. The conclusion must be that the photoperiodic mechanism in these examples is a heavily dampened oscillator, presenting properties of an hourglass-like timer, but measuring nightlength in natural, $24 \mathrm{~h}$ cycles according to the principles of external coincidence.

\section{Experimental and theoretical analysis of External Coincidence in Sarcophaga argyrostoma}

In external coincidence light has two functions: firstly, it entrains the photoperiodic oscillator to the light-dark cycle by inducing phase shifts, either advances or delays, and secondly, it controls the switch between diapause and nondiapause development by interacting with the photoinduc- ible phase $\left(\varphi_{\mathrm{i}}\right)$. This suggests that further analysis of the photoperiodic phenomenon should include study of phase changes elicited by experimental lighting regimes. Since the photoperiodic oscillator remains hidden, however, further progress was dependent on using an overt rhythm as 'hands of the clock' - following Bünning (1960) who used up-and-down movements of bean seedling leaves for this purpose. This experimental approach was adopted for $S$. argyrostoma using the rhythm of adult eclosion as an indicator of phase (Saunders, 1978a), the success of this procedure depending on the two phenomena - eclosion and diapause induction - being sufficiently similar in most of their basic circadian properties.

Phase shifts engendered by single 12-h light pulses (240 $\mu \mathrm{W} \mathrm{cm}^{-2}$ ) interrupting darkness during the photoperiodic 'sensitive period' (from first instar larvae to the point at which mature larvae enter the soil to form puparia) were used to construct an 'extended' PRC for the eclosion rhythm (Fig. 9). Parental flies were raised in continuous light (LL) and when first instar larvae were deposited on the meat, cultures were transferred to darkness (DD), the phase of the oscillation at the LL/DD transition being equivalent to CT 12 h (Pittendrigh, 1966; Peterson \& Saunders, 1980). Phase shifts caused by the $12 \mathrm{~h}$ light pulses during the first day or two gave high-amplitude Type 0 responses which then steadily declined through a series of lower-amplitude Type 1 PRCs to final extinction when the larvae formed puparia, this reduction in magnitude of the phase shifts mirroring presumed dampening of the photoperiodic oscillation (Saunders, 1979a). Cultures of larvae transferred 
from LL to DD on days 2, 3 and 5 of larval development, and then tested with $12 \mathrm{~h}$ light pulses, showed initial boosting of response to Type $0 \mathrm{PRCs}$, but then a rapid decline to Type 1 (Saunders, 1978a). Type 0 PRCs (Winfree, 1970) induced by $12 \mathrm{~h}$ light pulses during early larval development (photoperiodically the most sensitive period) were sufficient to set the clock to a phase relationship close to its final value; the following Type 1 PRCs with reduced phase shifts were then sufficient to maintain that relationship by 'fine tuning'.

In order to proceed further, a 'family' of eclosion rhythm PRCs was then constructed for light pulses with duration between 1 and $20 \mathrm{~h}$, applied during the first $24 \mathrm{~h}$ of larval life, the time when larvae were maximally sensitive to photoperiod. Pulses of 1 to $3 \mathrm{~h}$, and probably also $4 \mathrm{~h}$, of white light $\left.(240 \mu \mathrm{W} \mathrm{cm})^{-2}\right)$ were found to elicit low-amplitude Type 1 PRCs during the first subjective night whereas those of $5 \mathrm{~h}$ or more induced higher-amplitude Type 0 PRCs (Saunders, 1978a). These data were then used in a computer program designed to calculate approaches to steady state entrainment in a wide range of experimental light cycles performed to investigate phase relationships between the photoinducible phase $\left(\varphi_{\mathrm{i}}\right)$ and the light (see below). Since the critical nightlength in S. argyrostoma was shown to be $9.5 \mathrm{~h}, \varphi_{\mathrm{i}}$ was calculated to lie at about CT $12+9.5 \mathrm{~h}$, or at CT $21.5 \mathrm{~h}$. Results of this experimental procedure, therefore, could be used to determine whether $\varphi_{\mathrm{i}}$ - a phase centred at CT $21.5 \mathrm{~h}$, but presumably wider - was illuminated (or not) and these data compared with rates of diapause induction. And since the circadian period of the eclosion rhythm was very close to $24 \mathrm{~h}$, these calculations could be conducted in 'real time'.

This experimental procedure was applied to cultures exposed to a wide range of lighting cycles including skeleton photoperiods, night interruption and Nanda-Hamner experiments (Saunders, 1978a, 1979b). Cultures of first instar larvae were transferred from LL into DD (phase $=$ CT $12 \mathrm{~h}$ ). Phase shifts caused by each light pulse in the sequence of light pulses during the photoperiodic sensitive period were then computed noting whether or not $\varphi_{\mathrm{i}}$ coincided with (or came close to) illumination by a main light component or a scanning pulse. In practically every case diapause incidence was found to be high when the photoinducible phase fell in the dark, but low when it coincided with, or came very close to, a short light pulse or a longer light component, providing strong evidence for external coincidence as the basis for photoperiodic induction in S. argyrostoma.

This conclusion was particularly compelling in cycles comprising $1 \mathrm{~h}$ light pulses in cycle lengths $(\mathrm{T} h)$ ranging from $\mathrm{T}=21 \mathrm{~h}(1 \mathrm{~L}: 20 \mathrm{D})$ to $\mathrm{T}=30.5 \mathrm{~h}(1 \mathrm{~L}: 29.5 \mathrm{D})$ encompassing the primary range of entrainment of the presumed photoperiodic oscillation (Saunders, 1979b). In 'T-experiments' of this type, Pittendrigh $(1965,1966)$ showed that when $\mathrm{T}$ is greater than the period of the oscillation $(\tau \mathrm{h})$ the 1-h light pulse must come to lie in the early subjective night (CT 12 to $18 \mathrm{~h}$ ) to cause phase delays in each cycle (to correct $\tau$ to $T$ ) whereas when $T$ is less than $\tau$, the pulse must fall in the late subjective night (CT 18 to $24 \mathrm{~h}$ ) to

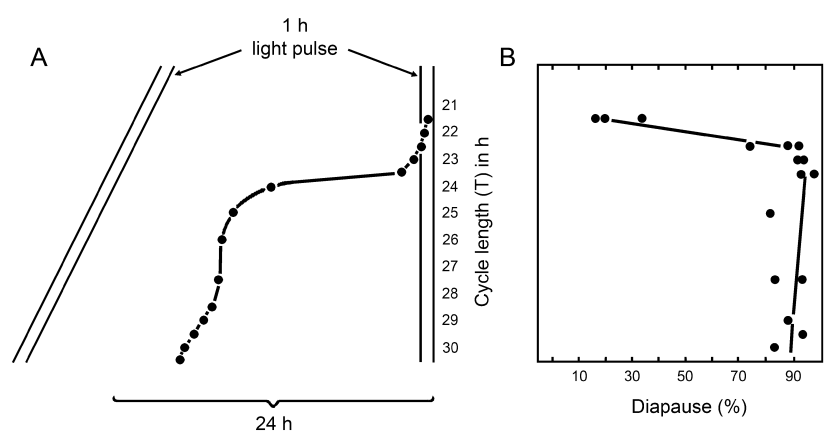

Fig. 10. Sarcophaga argyrostoma. A - computed phase relationships of the photoinducible phase ( $\varphi_{i}$ at CT $21.5 \mathrm{~h}$ ) to repeated $1 \mathrm{~h}$ pulses of light in cycles ranging in length from $21.5 \mathrm{~h}(1 \mathrm{~L}: 20.5 \mathrm{D})$ to $30.5 \mathrm{~h}(1 \mathrm{~L}: 29.5 \mathrm{D})$ across the primary range of entrainment for $1 \mathrm{~h}$ pulses. B - diapause induction in these cycles, showing that when the photoinducible phase falls in the dark diapause incidence is high, but when it coincides with, or comes close to the $1 \mathrm{~h}$ light pulse, it is reduced. (From Saunders, 1979b.)

cause the necessary phase advances. Just by altering cycle length in these $\mathrm{T}$ cycles, therefore, a short light pulse can be made to illuminate different circadian phases. Results of this experiment (Fig. 10) showed that when T was greater than $\tau$ (and $\varphi_{\mathrm{i}}$ computed to fall in the dark) diapause incidence was high, whereas when $T$ was less than $\tau$ (and $\varphi_{i}$ computed to coincide with, or come close to, the $1 \mathrm{~h}$ pulse of light) diapause incidence was greatly reduced.

Further evidence consistent with external coincidence in flesh flies has since been obtained in action spectrum studies in S. similis (Goto \& Numata, 2009). Like earlier studies with $M$. viciae (Lees, 1973), maximum long-day response to light pulses early in the scotophase (at point A of night interruption experiments) was observed in the blue-green (470 to $583 \mathrm{~nm}$ ) region of the spectrum, perhaps indicating entrainment by CRYPTOCHROME, whereas a wider response up to and including red (395 to $660 \mathrm{~nm}$ ) was observed for light pulses late in the night (at point B), suggesting that coincidence of light with $\varphi_{i}$ involves an additional photoreceptor, possibly opsin-based and absorbing longer wavelength light. Also, working with latitudinal strains of S. similis, Yamaguchi \& Goto (2019) showed that $\varphi_{\mathrm{i}}$ occurred earlier in the night in flies from more northerly locations, consistent with the shorter critical night length observed at these higher latitudes. In addition, Yamamoto et al. (2017) demonstrated systematic changes in PERIODimmunoreactivity in cerebral neurons of $S$. similis larvae raised in long (12L: 12D) and short (16L : 8D) nights, suggesting that period and its protein PER may be constituents of the photoperiodic clock in this species.

\section{The Internal Coincidence alternative: Dawn and dusk oscillators}

Although external coincidence (Fig. 4) is an appropriate model for the photoperiodic clock in flesh flies it may be less appropriate for some other species, suggesting that different circadian-based clocks probably exist - such as internal coincidence in which the clock comprises (at least) two oscillators, one a 'morning' component phaseset by 'dawn' and a second or 'evening' component phase- 
set by 'dusk' (e.g. Tyshchenko, 1966; Pittendrigh, 1972). Data from Nanda-Hamner experiments with $N$. vitripennis (Fig. 3a), for example, revealed circadian involvement in the photoperiodic response (Saunders, 1974), but did not easily lend themselves to interpretation in terms of simple external coincidence. In particular, the 'ascending slopes' of the high diapause 'mountains' in an extended 'circadian surface' (Fig. 6b) appeared to be parallel to dusk whereas the 'descending slopes' appeared to lie parallel to dawn, initially suggesting an interaction between two oscillators, as in the internal alternative.

Additional evidence, initially in favour of an internal coincidence type of clock in $N$. vitripennis, was then obtained from two further experiments. The first of these demonstrated that the diapause-nondiapause switch may be regulated by daily thermoperiod in the complete absence of light (Saunders, 1973b), and the second showing identical action spectra for 'long-day' nondiapause development caused by supplementary light pulses applied both early and late in the subjective night (Saunders, 1975). Results of these experiments, therefore, differed from comparable experiments with flesh flies (Saunders, 1978b, 1984; Goto \& Numata, 2009) underlining differences between the two species.

More complex versions of internal coincidence (often termed 'circadian resonance') are also theoretically possible (Pittendrigh, 1981; Saunders, 2016, 2020) but will not be considered further in this review.

\section{Future directions}

External coincidence is an adequate representation of the photoperiodic clock in flesh flies. It may also represent the clock in other species, particularly for holometabola in which diapause occurs in larvae and pupae, and in those studies suggesting a light-sensitive photoinducible phase and probable brain-centred photoreception (e.g. Bowen et al., 1984). It is also indicated in some hemimetabola such as the aphid $M$. viciae which present similar characteristics (Lees, 1964, 1973).

In flesh flies and other insects presenting external coincidence, further progress should include characterisation of the photoinducible phase: whether it is represented, for example, by opsin-based pigments in the larval brain, and how dawn light interacts with these putative photoreceptors and 'clock' neurons, ultimately regulating the release of prothoracicotropic hormone (PTTH) from the brain to initiate the PTTH-ecdysteroid cascade controlling diapause or nondiapause development (Richard \& Saunders, 1987; Richard et al., 1987). In S. argyrostoma the nature of the photoperiodic 'counter' mechanism remains obscure. What is the nature of the accumulating diapause-promoting product - the 'diapause titre' of Gibbs (1975), or the value INDSUM in the computer model of Lewis \& Saunders (1987) - and how is it transmitted through successive instars only to 'switch off' PTTH release before pupaladult differentiation (Richards et al., 1987)?

In $N$. vitripennis, further progress should include identification of photoreceptors in the compound eyes, perhaps by RNA-interference studies of opsin genes. The oscillatory (circadian) nature of the photoperiodic clock in Nasonia is also one of the least understood aspects of diapause induction. Early interpretation of the data reviewed above (Saunders, 1974) suggested that photoperiod was measured by seasonal changes in the phase relationship between two circadian oscillators, one phase set by dawn ('morning') and the other by dusk ('evening'), and that light had its role in entrainment - but not in photoinduction by temporal coincidence with a light-sensitive, or photoinducible phase. The conclusion that photoperiodic time measurement in Nasonia presents characteristics of internal coincidence therefore remains a possible interpretation. However, much of this evidence comes from Nanda-Hamner experiments which are difficult to interpret - and evidence from the Bünsow protocol (Fig. 2) seems to show a single light-sensitive phase during each subjective night and therefore more consistent with external than with internal coincidence. This divergence in interpretation poses the question: is the photoperiodic clock in N. vitripennis based on one oscillator (as in external coincidence), or on two (as in the internal alternative)?

In the Nasonia circadian surface (Fig. 6b), the three high diapause 'mountains' or peaks are evidence attesting to the underlying circadian nature of the photoperiodic response. Each of the three peaks, however, are compounded from several light-dark cycles. The first of these (peak a), centring around $24 \mathrm{~h}$, is a compound of all of the 14 or $1524-\mathrm{h}$ cycles encountered by the adult wasps before the larvae they produced were assessed for diapause or nondiapause development. The second (peak b), around $48 \mathrm{~h}$, is similarly a compound of the seven 48-h cycles encountered during the experiment, and the third (peak c) is a compound of only three 72-h cycles. Data contributing to peaks two and three, therefore, contain two or three cycles of DD freerun, respectively, during which time a photoperiodic oscillator may dampen.

In the first peak it is evident that diapause incidence rises abruptly about $9 \mathrm{~h}$ after light-off in cycles containing photophases longer than about $12 \mathrm{~h}$, thus showing a critical nightlength (CNL) as in 'natural' $24 \mathrm{~h}$ light cycles similar to that observed for $S$. argyrostoma (and external coincidence). This relationship to dusk is less well marked in the second peak. In the third peak it becomes apparent that diapause onset is becoming parallel to dawn rather than to dusk. The reason for this trend may be that, in these longer cycles, the oscillation dampens rapidly in darkness after each light pulse, weakening the 'strength' of the photophases to a point below which it is no longer phase-set to CT $12 \mathrm{~h}$ at the light-to-dark transition, leaving it to measure the passage of time from dawn rather than dusk. Therefore, although Nanda-Hamner experiments (Fig. 6b) may indicate internal coincidence in $N$. vitripennis, features of external coincidence are also apparent. Given that this wasp has become an important model species for studies in genetics, behaviour and ecology (Werren \& Loehlin, 2009), these complexities suggest that results using Nanda-Hamner experiments with Nasonia require further investigation. 
In conclusion, experimental approaches based on known circadian properties have provided strong evidence supporting Erwin Bünning's insightful proposition that photoperiodic time measurement is a function of the circadian system. Molecular investigations will undoubtedly produce stronger evidence for this association, but experiments such as those described in the present review, based on a comparative approach of photoperiodic timing in a circadian context, should further strengthen this connection and reveal important specific differences.

ACKNOWLEDGEMENTS. R.M.K. Saunders is thanked for assistance in figure production.

\section{REFERENCES}

AdKIsson P.L. 1964: Action of the photoperiod in controlling insect diapause. - Am. Nat. 98: 357-374.

AdKISSON P.L. 1966: Internal clocks and insect diapause. - Science 154: 234-241.

BAKER J.R. \& RANSON R.M. 1932: Factors affecting the breeding of the field mouse (Microtus agrestis). Part 1. - Proc. R. Soc. Lond. 110: 313-323.

BECK S.D. 1962: Photoperiodic induction of diapause in an insect. - Biol. Bull. 122: 1-12.

Blaney L.T. \& Hamner K.C. 1957: Inter-relations among the effects of temperature, photoperiod, and dark period on floral initiation of Biloxi soybean. - Bot. Gaz. 119: 10-24.

Bowen M.F., SAunders D.S., Bollenbacher W.E. \& Gilbert L.I. 1984: In vitro reprogramming of the photoperiodic clock in an insect brain-retrocerebral complex. - Proc. Natn. Acad. Sci. USA 81: 5881-5884.

BüNNING E. 1936: Die endogene Tagesrhythmik als Grundlage der Photoperiodischen Reaktion. - Ber. Dt. Bot. Ges. 54: 590-607.

BüNNING E. 1960: Circadian rhythms and time measurement in photoperiodism. - Cold Spring Harb. Symp. Quant. Biol. 25: 249-256.

BüNNING E. 1969: Common features of photoperiodism in plants and animals. - Photochem. Photobiol. 9: 219-228.

BüNSOw R.C. 1953: Uber Tages- und Jahresrhythmische Anderungen der Photoperiodischen Lichteropfindlichkeit bei Kalanchoë blossfeldiana und ihre Beziehungen zur endogenen Tagesrhythmik. - Z. Bot. 41: 257-276.

Chandrashekaran M.K. 2006: Erwin Bünning (1906-1990): A centennial homage. - J. Biosci. 31: 5-12.

Claes H. \& Lang A. 1947: Die Blütenbildung von Hyoscyamus niger in 48-stündigen Licht-Dunkel-Zyklen und in Zyklen mit aufgeteilten Lichtphasen. - Z. Naturforsch. (B) 2: 56-68.

Danilevskit A.S., Goryshin N.I. \& TyshChEnKo V.P. 1970: Biological rhythms in terrestrial arthropods. - Annu. Rev. Entomol. 15: 201-244.

Darwin C. \& Darwin F. 1880: The Power of Movement in Plants. John Murray, London, $593 \mathrm{pp}$.

De Mairan J.J. 1729: Observations botaniques. - Hist. Acad. Roy. Soc. 1729: 35-36.

Garner W.W. \& Allard H.A. 1920: Effects of the relative length of the day and night and other factors of the environment on growth and reproduction in plants. - J. Agric. Res. 18: 553606.

GiBBS D. 1975: Reversal of pupal diapause in Sarcophaga argyrostoma by temperature shifts after puparium formation. $-J$. Insect Physiol. 21: 1179-1186.

Gото S.G. 2013: Roles of circadian clock genes in insect photoperiodism. - Entomol. Sci. 16: 1-16.
Goto S.G. \& Numata H. 2009: Possible involvement of distinct photoreceptors in the photoperiodic induction of diapause in the flesh fly Sarcophaga similis. - J. Insect Physiol. 55: 401407.

Hall J.C. 2003: Genetics and molecular biology of rhythms in Drosophila and other insects. - Adv. Genet. 48: 1-280.

HAMNER K.C. 1960: Photoperiodism and circadian rhythms. Cold Spring Harb. Symp. Quant. Biol. 25: 269-277.

HAMNER W.M. 1963: Diurnal rhythms and photoperiodism in testicular recrudescence of the house finch. - Science 142: 1294-1295.

Hardin P.E. 2005: The circadian timekeeping system of Drosophila. - Curr. Biol. 15: R714-722.

Kauranan H., Kinnunen J., Hillos A.-L., Lankinen P., Hopkins D., Wiberg R.A.W., Ritchie M.G. \& Hoikkala A. 2019: Selection for reproduction under short photoperiods changes diapause-associated traits and induces widespread genomic divergence. - J. Exp. Biol. 222: 205831, 14 pp.

Kogure M. 1933: The influence of light and temperature on certain characters of the silk-worm, Bombyx mori. - J. Dept. Agr. Kyushu Univ. 4: 1-93.

Konopka R. \& Benzer S. 1971: Clock mutants of Drosophila melanogaster. - Proc. Natn. Acad. Sci. USA 68: 2112-2116.

LEES A.D. 1965: Is there a circadian component in the Megoura photoperiodic clock? In Aschoff J. (ed.): Circadian Clocks. North-Holland, Amsterdam, pp. 351-356.

LeEs A.D. 1966: The control of polymorphism in aphids. - Adv. Insect Physiol. 3: 207-277.

LEES A.D. 1971: The relevance of action spectra in the study of insect photoperiodism. In Menaker M. (ed.): Biochronometry. National Academy of Sciences, Washington, pp. 372-380.

LEEs A.D. 1973: Photoperiodic time measurement in the aphid Megoura viciae. - J. Insect Physiol. 19: 2279-2316.

LEES A.D. 1986: Some effects of temperature on the hour glass photoperiod timer in the aphid Megoura viciae. - J. Insect Physiol. 32: 79-89.

LEWIS R.D. \& SAUNDERS D.S. 1987: A damped circadian oscillator model of an insect photoperiodic clock. I. Description of the model based on a feedback control system. $-J$. Theor. Biol. 128: $47-59$.

Marcovitch S. 1923: Plant lice and light exposure. - Science 58: $537-538$.

MARCOVITCH S. 1924: The migration of the Aphididae and the appearance of sexual forms as affected by the relative length of daily light exposure. - J. Agric. Res. 27: 513-522.

Melchers G. 1956: Die Beteiligung der endonomen Tagesrhythmik am Zustandekommen der photoperiodischen Reaktion der Kurztagpflanze Kalanchoë blossfeldiana. - Z. Naturforsch. (B) 11: 544-548.

NANDA K.K. \& HAMNER K.C. 1958: Studies on the nature of the endogenous rhythm affecting photoperiodic response of Biloxi soybean. - Bot. Gaz. 120: 14-25.

Numata H. \& HidaKa T. 1983: Compound eyes as the photoperiodic receptors in the bean bug. - Experientia 39: 868-869.

Peterson E.L. \& Saunders D.S. 1980: The circadian eclosion rhythm in Sarcophaga argyrostoma: a limit cycle representation of the pacemaker. - J. Theor. Biol. 86: 265-277.

PITTENDRIGH C.S. 1965: On the mechanism of entrainment of a circadian rhythm by light cycles. In Aschoff J. (ed.): Circadian Clocks. North-Holland, Amsterdam, pp. 277-297.

PitTendRigh C.S. 1966: The circadian oscillation in Drosophila pseudoobscura pupae: a model for the photoperiodic clock. Z. Pflanzenphysiol. 54: 275-307. 
PittendRigh C.S. 1972: Circadian surfaces and the diversity of possible roles of circadian organization in photoperiodic induction. - Proc. Natn. Acad. Sci. USA 69: 2734-2737.

PITTENDRIGH C.S. 1981: Circadian organization and the photoperiodic phenomena. In Follett B.K. \& Follett D.E. (eds): Biological Clocks in Seasonal Reproductive Cycles. John Wright \& Sons, Bristol, pp. 1-35.

Richard D.S. \& Saunders D.S. 1987: Prothoracic gland function in diapause and nondiapause Sarcophaga argyrostoma and Calliphora vicina. - J. Insect Physiol. 33: 385-392.

Richard D.S., Warren J.T., Saunders D.S. \& Gilbert L.I. 1987: Haemolymph ecdysteroid titres in diapause and nondiapause destined larvae and pupae of Sarcophaga argyrostoma. $-J$. Insect Physiol. 33: 115-122.

RowAN W. 1926: On photoperiodism, reproductive periodicity and the annual migration of birds and certain fishes. - Proc. Soc. Nat. Hist. 38: 147-189.

SAUNDERS D.S. 1965: Larval diapause of maternal origin: Induction of diapause in Nasonia vitripennis (Walk.) (Hymenoptera: Pteromalidae). - J. Exp. Biol. 42: 495-508.

SAUNDERS D.S. 1966: Larval diapause of maternal origin - II. The effect of photoperiod and temperature on Nasonia vitripennis. — J. Insect Physiol. 12: 569-581.

SAUNDERS D.S. 1968: Photoperiodism and time measurement in the parasitic wasp, Nasonia vitripennis. - J. Insect Physiol. 14: 433-450.

SAUNDERS D.S. 1970: Circadian clock in insect photoperiodism. - Science 169: 601-603.

SAUNDERS D.S. 1971: The temperature compensated photoperiodic clock 'programming' development and pupal diapause in the flesh-fly, Sarcophaga argyrostoma. - J. Insect Physiol. 17: 801-812.

SAUNDERS D.S. 1973a: The photoperiodic clock in the flesh fly Sarcophaga argyrostoma. - J. Insect Physiol. 19: 1941-1954.

SAUNDERS D.S. 1973b: Thermoperiodic control of diapause in an insect: theory of internal coincidence. - Science 181: 358360

SAUNDERS D.S. 1974: Evidence for 'dawn' and 'dusk' oscillators in the Nasonia photoperiodic clock. - J. Insect Physiol. 20: $77-88$.

SAUNDERS D.S. 1975: Spectral sensitivity and intensity thresholds in Nasonia photoperiodic clock. - Nature 233: 732-734.

SAUNDERS D.S. 1978a: An experimental and theoretical analysis of photoperiodic induction in the flesh-fly, Sarcophaga argyrostoma. - J. Comp. Physiol (A) 124: 75-95.

SAUNDERS D.S. 1978b: Internal and external coincidence and the apparent diversity of photoperiodic clocks in insects. $-J$. Comp. Physiol. (A) 127: 197-207.

SAUNDERS D.S. 1979a: The circadian eclosion rhythm in Sarcophaga argyrostoma: delineation of the responsive period for entrainment. - Physiol. Entomol. 4: 263-274.

SAUNDERS D.S. 1979b: External coincidence and the photoinducible phase in the Sarcophaga photoperiodic clock. - J. Comp. Physiol. (A) 132: 179-189.

SAUNDERS D.S. 1981: Insect photoperiodism: the clock and the counter. - Physiol. Entomol. 6: 99-116.

SAUNDERS D.S. 1982a: The effect of ultra-short photoperiods on the seasonal clock in Sarcophaga argyrostoma. - J. Comp. Physiol. (A) 145: 421-429.

SAUNDERS D.S. 1982b: Photoperiodic induction of pupal diapause in Sarcophaga argyrostoma: temperature effects on circadian resonance. - J. Insect Physiol. 28: 305-310.

SAUNDERS D.S. 1984: Photoperiodic time measurement in Sarcophaga argyrostoma: An attempt to use daily temperature cycles to distinguish external from internal coincidence. $-J$. Comp. Physiol. (A) 154: 789-794.

SAUNDERS D.S. 1987a: Insect photoperiodism: the linden bug, Pyrrhocoris apterus, a species that measures daylength rather than nightlength. - Experientia 43: 935-937.

SAUNDERS D.S. 1987b: Maternal influence on the incidence and duration of larval diapause in Calliphora vicina. - Physiol. Entomol. 12: 331-338.

SAUNDERS D.S. 1992: The photoperiodic clock and "counter" in Sarcophaga argyrostoma: experimental evidence consistent with "external coincidence" in insect photoperiodism. $-J$. Comp. Physiol. (A) 170: 121-127.

SAUNDERS D.S. 2002: Insect Clocks. $3^{\text {rd }}$ ed. Elsevier, Amsterdam, $560 \mathrm{pp}$.

SAUNDERS D.S. 2013: Insect photoperiodism: Measuring the night. - J. Insect Physiol. 59: 1-10.

SAUNDERS D.S. 2016: The formal 'structure' and function of the insect photoperiodic clock: a tribute to Colin S. Pittendrigh. Physiol. Entomol. 41: 1-18.

SAunders D.S. 2020: Dormancy, diapause, and the role of the circadian system in insect photoperiodism. - Annu. Rev. Entomol. 65: 19.1-19.17.

SAUNDERS D.S. \& Bertossa R.C. 2011: Deciphering time measurement: the role of circadian 'clock' genes and formal experimentation in insect photoperiodism. - J. Insect Physiol. 57: 557-566.

SAUNDERS D.S. \& LewIS R.D. 1987a: A damped circadian oscillator model of an insect photoperiodic clock. II. Simulations of the shapes of the photoperiodic response curves. $-J$. Theor. Biol. 128: 61-71.

SAUNDERS D.S. \& LEwIS R.D. 1987b: A damped circadian oscillator model of an insect photoperiodic clock. III. Circadian and "hourglass" responses. - J. Theoret. Biol. 128: 73-85.

SAUNDERS D.S. \& LEWIS R.D. 1988: The photoperiodic clock and counter mechanism in two species of flies: evidence for damped circadian oscillators in time measurement. - J. Comp. Physiol. (A) 163: 365-371.

TAKImOTo A. \& HAMNER K.C. 1964: Effect of temperature and preconditioning on photoperiodic response of Pharbitis nil. Plant Physiol. 39: 1024-1030.

TYSHCHENKo V.P. 1966: Two-oscillatory model of the physiological mechanism of insect photoperiodic reaction. - Zhur. Obshch. Biol. 27: 209-222 [in Russian].

Tyukmaeva V., Lankinen P., Kinnunen J., Kauranan H. \& HoikKALA A. 2020: Latitudinal clines in the timing and temperaturesensitivity of photoperiodic diapause in Drosophila montana. - Ecography 43: 1-11.

Vaz Nunes M. \& SAunders D.S. 1999: Photoperiodic time measurement in insects: a review of clock models. $-J$. Biol. Rhythms 14: 84-104.

VAZE K.M. \& Helfrich-Förster C. 2016: Drosophila ezoana uses an hour-glass or highly damped circadian clock for measuring night length and inducing diapause. - Physiol. Entomol. 41: $378-389$.

Werren J.H. \& Loehlin D.W. 2009: The parasitoid wasp Nasonia: an emerging model species with haploid male genetics. Cold Spring Harb. Protoc. 10: 134, 31 pp.

WINFREE A.T. 1970: Integrated view of resetting a circadian clock. - J. Theor. Biol. 28: 327-374.

YAMAGUChI K. \& Gото S.G. 2019: Distinct physiological mechanisms induce latitudinal and sexual differences in the photoperiodic induction of diapause in a fly. $-J$. Biol. Rhythms 34: 293-306. 
Yамамото M., Shiga S. \& Goto S.G. 2017: Distribution of PERIOD-immunoreactive neurons and temporal change of the immunoreactivity under long-day and short-day conditions in the larval brain of the flesh fly Sarcophaga similis. - Chronobiol. Internat. 34: 819-825.
ZINN J.G. 1759: Von dem Schlafe der Pflanzen. - Hamburgisches Magazin 22: 40-50.

Received November 30, 2020; revised and accepted January 6, 2021 Published online January 21, 2021 